\title{
Extraction of Plastic Properties of Aluminum Single Crystal Using Berkovich Indentation
}

\author{
Jui-Chao Kuo* and I-Hua Huang \\ Department of Materials Science and Engineering, National Cheng Kung University, \\ No.1, University Road, Tainan 701, Taiwan, R. O. China
}

An alternative to extract representative stress-strain curves from a set of indentation data measured with the Berkovich indenter is developed in this study. The "yield" depth in the representative strain is identified by a double logarithm plot of indentation data, and it enables the establishment of a representative stress-strain curve. Comprehensive analyses are demonstrated using the new method proposed to capture the yield strength and the strain hardening exponent for an aluminum single crystal. [doi:10.2320/matertrans.M2010132]

(Received April 20, 2010; Accepted August 24, 2010; Published October 14, 2010)

Keywords: nanoindentation, representative strain, flow curve, pop-in

\section{Introduction}

Compared with mechanical properties tests, the nanoindentation technique is a convenient approach to characterize the nano-mechanical properties of surfaces and thin films. A number of analysis methods based on experimental and numerical investigations have been conducted on many materials to extract the mechanical properties from indentation load-displacement curves. During loading, the typical load-displacement response generally follows the relation described by Kick's Law, that is, $P=C h^{2}$ with loading curvature $C$. Based on Kick's Law, mechanical properties are obtained from the measured force-displacement curves such as elastic modulus, yield strength, strain hardening, residual stress, and creep behaviors. ${ }^{1-16)}$ The applications of the stress-strain curves can be found in describing the plastic behavior of materials and providing the materials parameters for deformation simulation.

A number of previous investigations were carried out, which prescribe the relationship between the measured loaddisplacement response and the mechanical properties by applying dimensional analysis using dimensionless $\Phi$ and $\Pi$ functions, which were introduced by Casals. ${ }^{17)}$ In the former case of the dimensionless $\Phi$ function, it provides the relationship between pressure $\bar{p}$ and the mechanical properties. In the latter case, the dimensionless $\Pi$ function relates the parameters from the $P-h$ curve to the mechanical properties.

Tabor ${ }^{1)}$ first proposed the relationship between representative stress and the mean pressure $\bar{p}$, as well as the definition of representative strain. Hence, the tensile stress-strain curve can be derived from indentation tests. A linear relationship between hardness $H$ and representative stress $\sigma_{\mathrm{r}}$ is proposed by Tabor ${ }^{18)}$ as follows:

$$
\sigma_{\mathrm{r}}=H / C
$$

The ratio $C$ of the hardness $H$ to the representative stress $\sigma_{\text {r }}$ is equal to 3 for Vickers indentations on metals, while the representative strain $\varepsilon_{\mathrm{r}}$ is between 0.08 and 0.1 . The hardness

*Corresponding author, E-mail: jckuo@mail.ncku.edu.tw of materials is defined as the ratio between the applied force $P$ and the projected contact area $A$ :

$$
H=P / A
$$

Based on Tabor's definition, the representative strain for a conical indenter with an indenter angle $\theta$, as shown in Fig. 1(a), is given by the following:

$$
\varepsilon_{\mathrm{r}}=0.2 \cot \theta
$$

The representative strain can be expressed in a simple relationship for a spherical indenter: ${ }^{19)}$

$$
\varepsilon_{\mathrm{r}}=0.2 a / R
$$

where $a$ represents the contact radius, and $R$ is the radius of the indenter (see Fig. 1(b)).

In this study, we follow the dimensionless $\Phi$ functions and propose a new analysis method for extracting the stress-strain curves from the measured data set of load and displacement for Berkovich indentation.

\section{Experiment}

A single crystal of $99.9999 \%$ pure aluminum was produced by the modified Bridgman method and then prepared by an electric discharge machine into sample geometry of $3 \times 12 \times 12 \mathrm{~mm}^{3}$. The orientation of the $\mathrm{Al}$ single crystal is $\left(265^{\circ}, 102^{\circ}, 190^{\circ}\right)$ in terms of Euler angles according to Bunge's definition, i.e., the out-of-plane direction parallel to [ $\overline{1} \overline{5} \overline{1}]$ which is close to [001]. One of the sample surfaces was obtained through grinding with 2000 grit $\mathrm{SiC}$ abrasive paper, then polishing with $0.5 \mu \mathrm{m}$ diamond paste. After polishing, the sample surface was electropolished to avoid the undesirable effects caused by mechanical hardening on the sample surface during sample preparation. The electropolishing process was in an electrolyte composed of $80 \%$ alcohol and $20 \%$ perchloric acid at room temperature under $20 \mathrm{~V}$ for $60 \mathrm{~s}$.

Nanoindentation was carried out using the MTS System Nano Indenter ${ }^{\circledR}$ XP which has a maximum load capability of $500 \mathrm{mN}$. A Berkovich diamond tip having a nominal edge radius of $20 \mathrm{~nm}$ was used. The parameters for these tests 
(a)

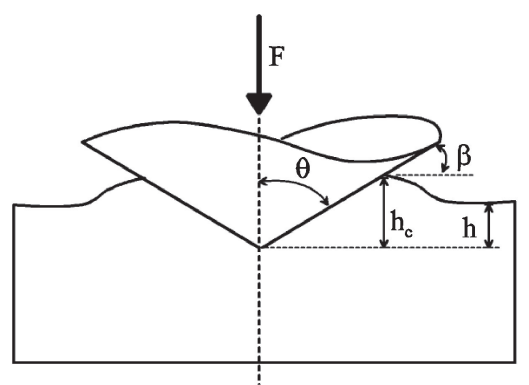

(b)

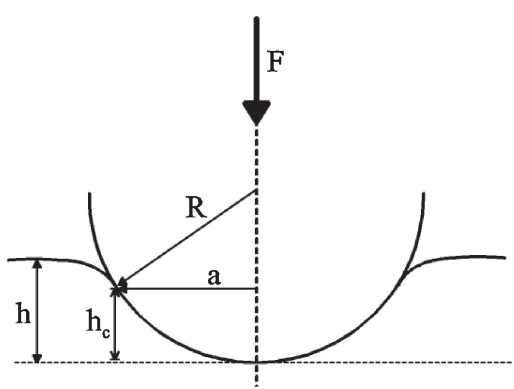

Fig. 1 Schematic illustrations of the indentation process with (a) a conical tip and (b) a spherical tip.

(a)

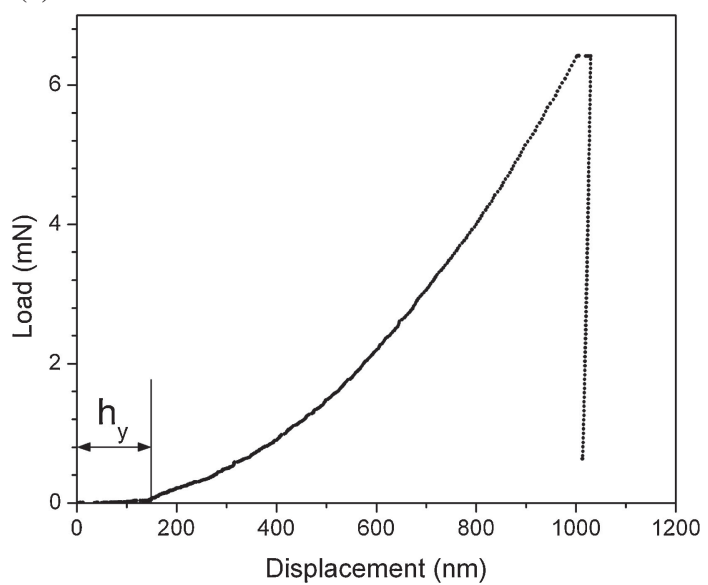

(b)

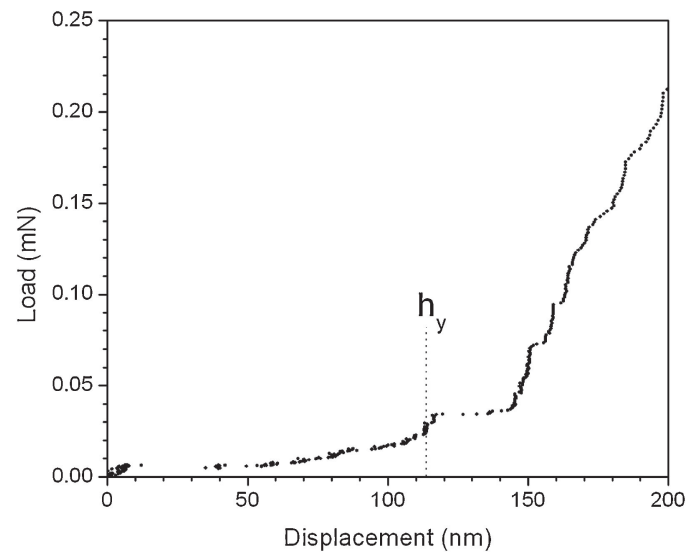

Fig. 2 (a) A load-displacement curve aluminum single crystal measured by Berkovich indentation with the CSM mode and (b) the magnification of the load-displacement curve from (a).

included a maximum penetration depth of $1000 \mathrm{~nm}$, a frequency target of $45 \mathrm{~Hz}$, a harmonic displacement target of $2 \mathrm{~nm}$, and a strain rate target of $0.050 \mathrm{~s}^{-1}$. Here, the CSM mode multiple indentations were performed on an aluminum single crystal. It is possible to obtain hardness and modulus continuously during loading by superimposing a small oscillation on the loading signal. This is known as continuous stiffness monitoring (CSM). For the aluminum single crystal, Fig. 2 shows a typical load-displacement curve obtained from Berkovich indentation.

\section{Extracting Representative Strain}

Analogous to the definition of "true strain" the strain used here can be expressed:

$$
\varepsilon=\int_{h_{0}}^{h} d \ln h=\ln h / h_{0}
$$

where $h_{0}$ is the initial depth and $h$ a given depth at a given time.

Therefore, the total strain $\varepsilon$ is composed of an elastic strain $\varepsilon_{\mathrm{e}}$ and a plastic strain $\varepsilon_{\mathrm{r}}$ (called representative strain) by:

$$
\begin{aligned}
\varepsilon & =\varepsilon_{\mathrm{e}}+\varepsilon_{\mathrm{r}} \\
& =\int_{h_{0}}^{h_{\mathrm{y}}} d \ln h+\int_{h_{\mathrm{y}}}^{h} d \ln h \\
& =\ln h_{\mathrm{y}} / h_{0}+\ln h / h_{\mathrm{y}}
\end{aligned}
$$

where $h_{0}$ is the initial depth and $h_{\mathrm{y}}$ the depth at yielding. Therefore, the representative strain $\varepsilon_{\mathrm{r}}$ can be expressed:

$$
\varepsilon_{\mathrm{r}}=\ln h / h_{\mathrm{y}}
$$

And then the indentation stress was adopted as

$$
\sigma_{\mathrm{r}}=P / A
$$

where $P$ is the applied force and $A$ the projected contact area. In this study the representative stress-strain curve was obtained using eq. (7) and eq. (8).

During indentation, the average pressure $\bar{p}$ according to the dimensionless function ${ }^{20)}$ can be given by:

$$
\bar{p}=\Phi \cdot \sigma_{y}
$$

where $\sigma_{y}$ is the yield stress and $\Phi$ is a dimensionless function. According to a power-law strain-hardening model, the plastic behavior of pure metals and alloys can be closely approximated in Fig. 3 and the true stress-strain relationship, if $\sigma \geq \sigma_{y}$, is given:

$$
\sigma_{\mathrm{r}}=K \varepsilon_{\mathrm{r}}^{n}
$$

where $K$ is the strength coefficient, $n$ is the strain hardening exponent, $\sigma_{\mathrm{r}}$ is the representative stress, and $\varepsilon_{\mathrm{r}}$ is the representative strain.

Substitution of eq. (10) into eq. (9) yields the following:

$$
\bar{p}=\Phi \cdot K \cdot \varepsilon_{\mathrm{r}}^{n}
$$

Substitution of eq. (7) into eq. (11) yields: 


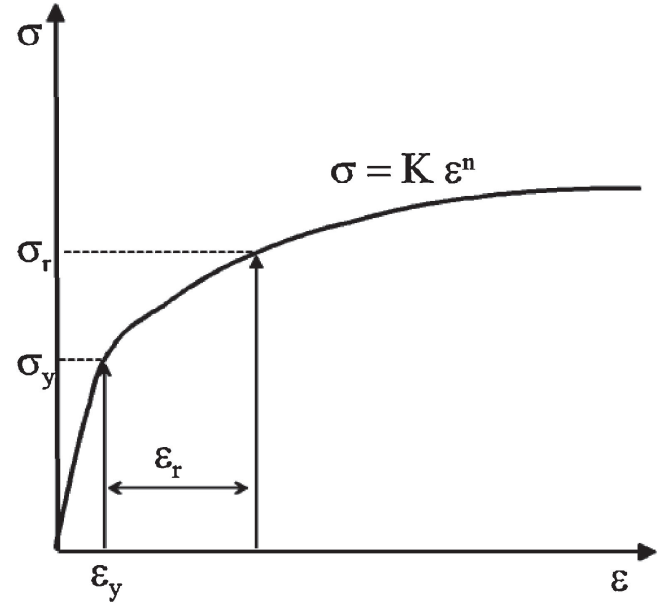

Fig. 3 A schematic illustration of the uniaxial true stress-strain curve of a power-law strain-hardening material with the representative strain $\varepsilon_{\mathrm{r}}$ and representative stress $\sigma_{\mathrm{r}}$ used in this study.

$$
\bar{p}=P / A=\Phi \cdot K \cdot\left(\ln h / h_{\mathrm{y}}\right)^{n}
$$

The relationship will be applied to extract the stress-strain curve from a load-displacement curve in this study.

The next step is to determine the "yield depth" $h_{\mathrm{y}}$ from raw data of load and displacement. Equation (12) can be transformed into a logarithm relationship:

$$
\ln \bar{p}=\ln \Phi K+n \ln \left(\ln h-\ln h_{\mathrm{y}}\right)
$$

where $\bar{p}$ is a average pressure, $K$ is the strength coefficient, $n$ is the strain hardening exponent, $h$ is the indentation depth, and $h_{\mathrm{y}}$ is the "yield" depth.

Differentiating eq. (13), the strain-hardening exponent of $n$ is obtained by:

$$
n=d(\ln \bar{p}) / d\left[\ln \left(\ln h-\ln h_{\mathrm{y}}\right)\right]
$$

Therefore, the strain-hardening exponent of $n$ in eq. (14) can be rewritten as follows:

$$
n=d \ln \bar{p} / d(\ln (\ln h)) \cdot\left[1-\left(\ln h_{\mathrm{y}} / \ln h\right)\right]
$$

The term of $\left(\ln h_{\mathrm{y}} / \ln h\right)$ is smaller than 1 because the indentation depth $h$ is larger than the "yield" depth $h_{\mathrm{y}}$ for $\sigma \geq \sigma_{y}$. In addition, it is well known that the strain-hardening exponent of $n$ is larger than zero. This implies that the slope of the double logarithm plot, $n$, should be larger than zero, that is, the slope of $d \ln \bar{p} / d(\ln (\ln h))$ should be positive. From the double logarithm plot it is can be divided into two regions, one is $d \ln \bar{p} / d(\ln (\ln h))>0$ in the region I and the other $d \ln \bar{p} / d(\ln (\ln h))<0$ in the region II. There exists a minimum point in the double which is taken as the "yield" depth. After that the representative strain is determined according to eq. (7).

Figure 4 shows, for instance, the double logarithm plot of $\ln \sigma_{\mathrm{r}}$ and $\ln \ln h$ as obtained from the load displacement in Fig. 3 for an aluminum single crystal. From the double logarithm plot in Fig. 4 it is can be observed two regions, region I with $d \ln \bar{p} / d(\ln (\ln h))>0$ and region II with $d \ln \bar{p} / d(\ln (\ln h))<0$. Assuming the strain-hardening exponent of $n>0$, it leads to the slope of $d \ln \bar{p} / d(\ln (\ln h))>0$ and this suggests that there exists a minimum point in the

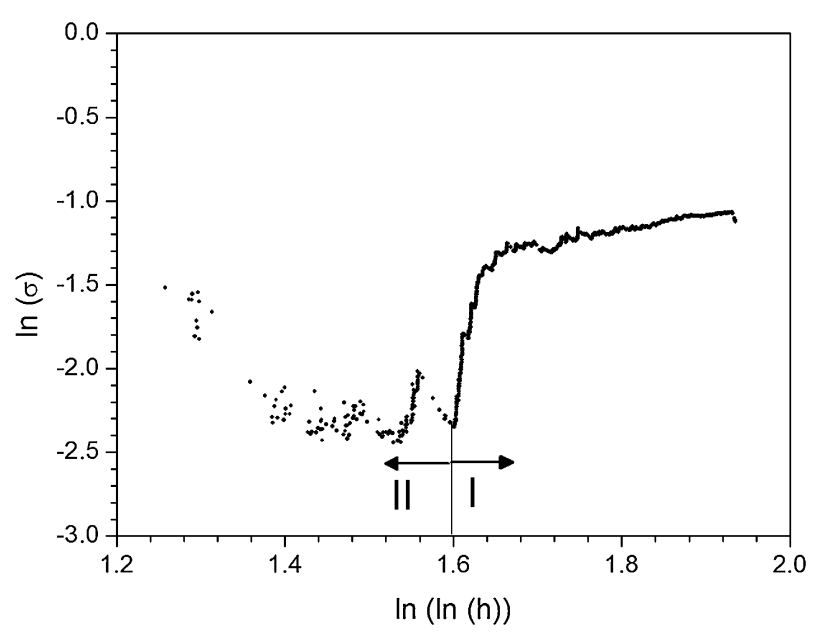

Fig. 4 Double-logarithm plot of $\ln \sigma_{\mathrm{r}}$ related to $\ln \ln h$ of aluminum.

double logarithm plot at $\ln \sigma_{\mathrm{r}}$ is of 1.56 as seen in Fig. 4. The value of $\ln \ln h$ at the minimum point corresponds to the indentation depth of $114.04 \mathrm{~nm}$ which is taken as the "yield" depth. At the "yield" depth $114.04 \mathrm{~nm}$ the slope of $d \ln \bar{p} / d(\ln (\ln h))$ is zero and therefore, the strain-hardening exponent of $n$ is also zero according to eq. (15). The physical meaning of the "yield" depth $h_{\mathrm{y}}$ corresponds to the depth at yielding as shown in Fig. 3. After the determination of the yield depth $h_{\mathrm{y}}$, the representative strain and stress were obtained using eq. (7) and eq. (8), respectively.

\section{Mechanical Property and Representative Stress- Strain Curve}

Using the data analysis method proposed by Oliver and Pharr, ${ }^{3)} E_{\mathrm{r}}$, which is the reduced modulus of materials, can be calculated by

$$
E_{\mathrm{r}}=\sqrt{\pi} / 2 \cdot S / \sqrt{A}
$$

where $S$ is elastic contact stiffness, and $A$ is the projected contact area. The elastic modulus $E$ of material can be calculated by:

$$
1 / E_{\mathrm{r}}=1-v^{2} / E+1-v_{i}^{2} / E_{i}
$$

where $E$ and $v$ are Young's modulus and Poisson's ratio of the indented material respectively; and $E_{i}$ and $v_{i}$ are the corresponding values of the indenter tip.

According to the method proposed by Oliver and Pharr, ${ }^{3)}$ the elastic modulus of the Al single crystal determined from the three load-displacement curves in Fig. 6(a) is in the range of 67.2-69.5 GPa and in good agreement with values reported in the literature. ${ }^{21,22)}$ At the indentation depth less than $200 \mathrm{~nm}$, a "staircase"-shaped $P-h$ curve which is called displacement burst (or "pop-in") ${ }^{23-25)}$ is shown in Fig. 3(b). The first displacement burst typically occurs at a load $P$ that corresponds to the maximum shear stress under the indenter which reaches the theoretical shear strength of the indented specimen material. Thus, the mechanism for this pop-in is due to the nucleation and propagation of dislocations. ${ }^{26)} \mathrm{Nix}$ and $\mathrm{Gao}^{27)}$ has proposed that the geometrically necessary dislocations created in small indentations create a hardening effect that is applied to explain this pop-in phenomenon. 
(a)

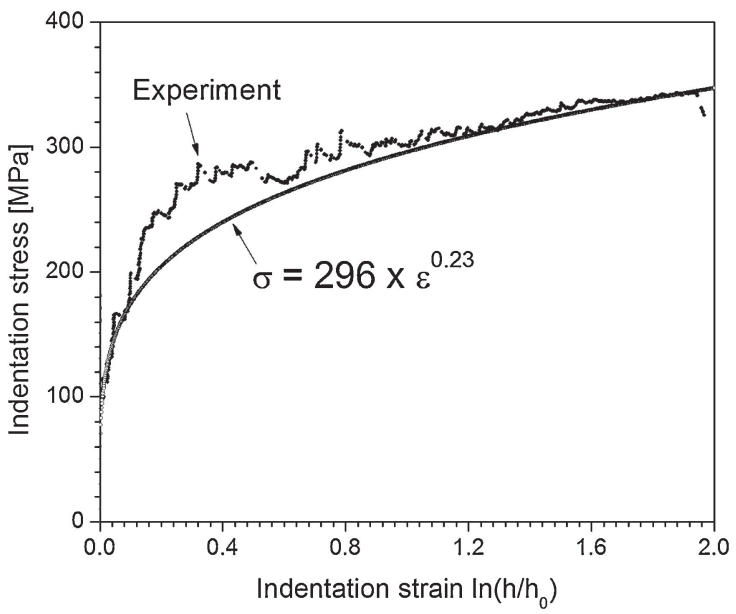

(b)

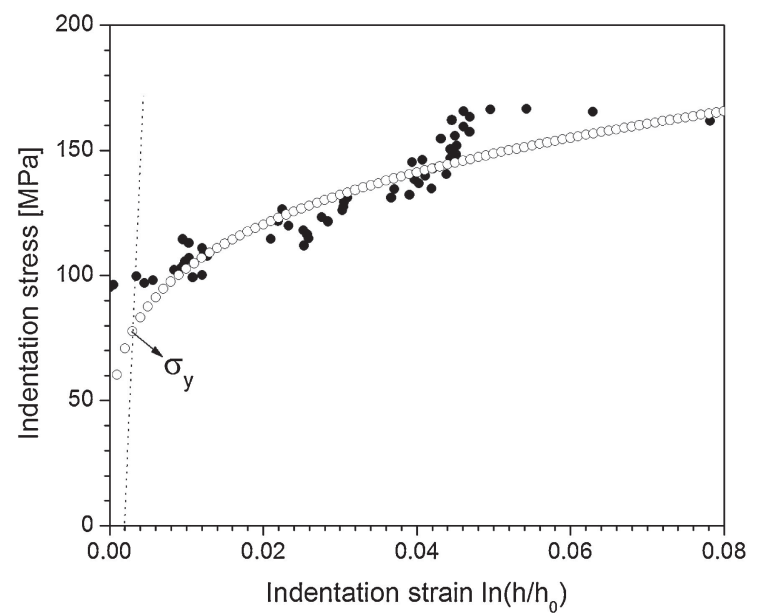

Fig. 5 (a) The stress-strain curves of an aluminum single crystal obtained with the method proposed by the present work and fitted with a power law. (b) The magnification of the load-displacement curve from (a).

(a)

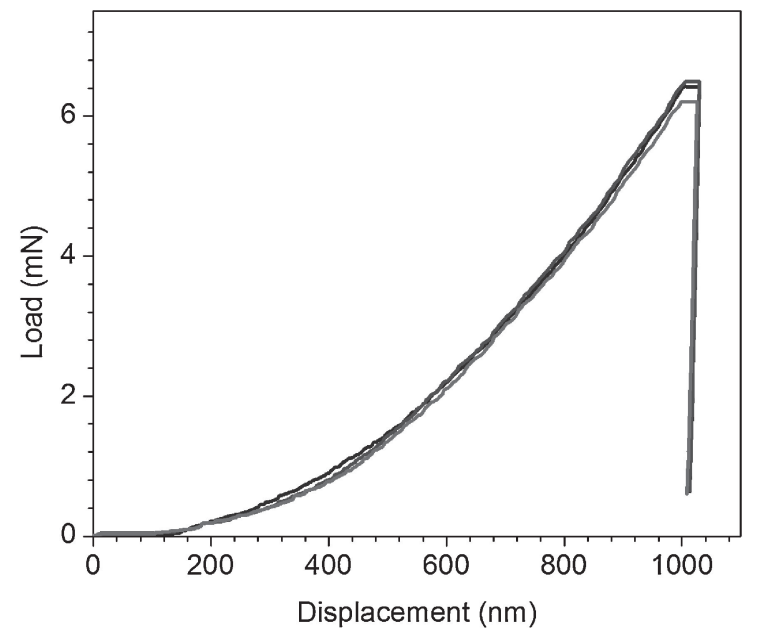

(b)

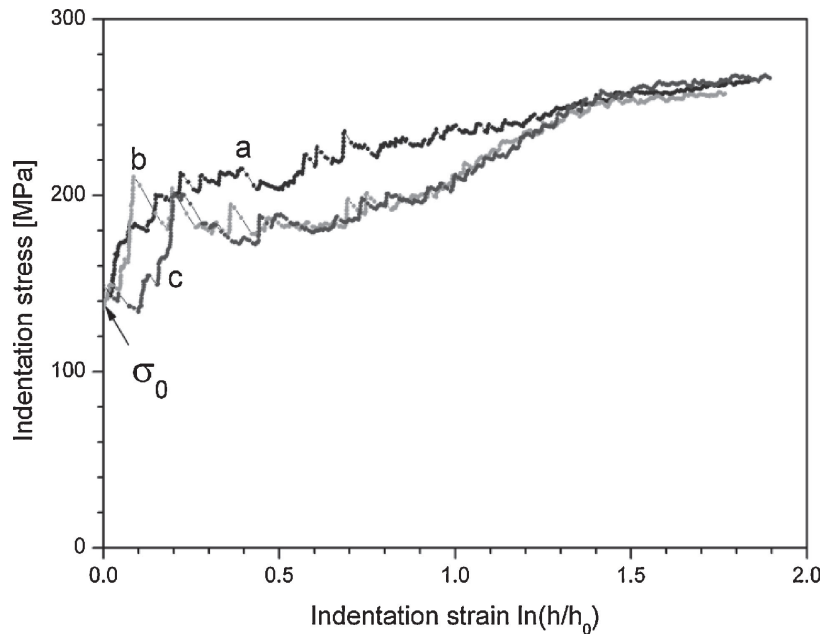

Fig. 6 (a) Three load-displacement curves of aluminum single crystal measured by Berkovich indentation with the CSM mode. (b) The corresponding stress-strain curves of an aluminum single crystal obtained from (a).

The indentation stress-strain curve was obtained for Berkovich indentation using the new methods proposed as shown in Fig. 5(a). The representative stress-strain curve in Fig. 5(a) appears "staircase"-shaped for the aluminum single crystal. It is observed that the three indentation stress-strain curves are not the same in Fig. 6(b), although the loaddisplacement curves are similar. This can be due to differences in local morphology in the nanoscale. Therefore, an indentation stress-strain curve was fitted using a power law. The strain-hardening exponent $n$ of 0.23 in Fig. 5(a) lies below the average value of the strain-hardening exponent between 0.285 and $0.31 .^{28-30)}$ Aluminum does not have a well-defined yield point, consequently, it is practice to define a yield stress for aluminum using the $0.2 \%$ offset method. The elastic modulus obtained from the method proposed by Oliver and Pharr ${ }^{3)}$ can be plotted in the indentation stressstrain curve and then the yield stress of $76.9 \mathrm{MPa}$ is able to be determined with the $0.2 \%$ offset method.

\section{Conclusions}

This new analysis procedure provided an alternative and easy approach proposed for establishing representative stress-strain curves for Berkovich tip. The representative strain is defined using the logarithm of the ratio of the total depth to the "yield" depth which corresponds to a minimum point in a double logarithm plot, that is, the depth at yielding. It is found that the strain-hardening exponent $n$ is 0.23 , and the yield stress is $76.9 \mathrm{MPa}$.

\section{Acknowledgements}

This research was sponsored by the National Science Council of Taiwan under Project No. NSC 96-2221-E-006124. The authors would like to thank Center for Micro/Nano Science and Technology for providing facilities. 


\section{REFERENCES}

1) M. F. Doerner and W. D. Nix: J. Mater. Res. 1 (1986) 601-609.

2) J. L. Loubet, J. M. Georges and G. Meille: Microindentation Techniques in Material Science and Engineering. Philadelphia ASTM STP 889, (Vickers Indentation Curves of Elastoplastic Materials 1986) pp. $72-89$.

3) W. C. Oliver and G. M. Pharr: J. Mater. Res. 7 (1992) 1564-1583.

4) M. Herrmann, N. Schwarzer, F. Richter, S. Fruhauf and S. E. Schulz: Surf. Coat. Technol. 201 (2006) 4305-4310.

5) J. S. Field and M. V. Swain: J. Mater. Res. 8 (1993) 297-306.

6) J. S. Field and M. V. Swain: J. Mater. Res. 10 (1995) 101-112.

7) A. Bolshakov, W. C. Oliver and G. M. Pharr: J. Mater. Res. 11 (1996) 760-768.

8) K. Shinohara, K. Yasuda, M. Yamada and C. Kinoshita: Acta Metall. Mater. 42 (1994) 3909-3915.

9) J. H. Ahn and D. Kwon: J. Mater. Res. 16 (2001) 3170-3178.

10) T. W. Capehart and Y. T. Cheng: J. Mater. Res. 18 (2003) 827-832.

11) A. F. Bower, N. A. Fleck, A. Needleman and N. Ogbonna: Proc. Roy. Soc. A 441 (1993) 97-124.

12) B. Storåkers and P. L. Larsson: J. Mech. Phys. Solids 42 (1994) $307-$ 332.

13) Y. T. Cheng and C. M. Cheng: Surf. Coat. Technol. 133-134 (2000) 417-424.

14) C. A. Schuhe, T. G. Nieh and Y. Kawamura: J. Mater. Res. 17 (2002) 1651-1654.

15) P. M. Sargent and M. F. Ashby: Mater. Sci. Technol. 8 (1992) 594-601.
16) Y. T. Cheng and C. M. Cheng: Int. J. Solids Struct. 36 (1999) 12311243.

17) O. Casals and J. Alcalá: Acta Mater. 53 (2005) 3545-3561.

18) D. Tabor: The hardness of metals, (Oxford: Oxford University Press, London, 1951).

19) K. L. Johnson: Contact mechanics, (Cambridge: Cambridge University Press 1985).

20) Y. P. Cao and J. Lu: Acta Mater. 52 (2004) 1143-1153.

21) G. Simmons and H. Wang: Single crystal elastic constants and calculated aggregate properties: A Handbook 2nd ed., (MIT Press, Cambridge, Massachusetts, 1971).

22) J. R. Davis: Metals Handbook: Desk Edition, 2nd ed., (ASM International Materials Park, OH, 1998).

23) C. A. Schuh, J. K. Mason and A. C. Lund: Nature Mater. 4 (2005) 617621.

24) D. Chrobak, K. Nordlund and R. Nowak: Phys. Rev. Lett. 98 (2007) 045502.

25) R. Nowak, D. Chrobak, S. Nagao, D. Vodnick, M. Berg, A. Tukiainen and M. Pessa: Nature Nanotechnol. 4 (2009) 287-291.

26) A. Gouldstone, N. Chollacoop, M. Dao, J. Li, A. M. Minor and Y. LShen: Acta Mater. 55 (2007) 4015-4039.

27) W. D. Nix and H. Gao: J. Mech. Phys. Solids 46 (1998) 411-425.

28) J. W. Yoon, F. Barlat, J. J. Gracio and E. Rauch: Int. J. Plasticity 21 (2005) 2426-2447.

29) M. A. Meyers and K. K. Chawla: Mechanical behavior of materials, (Prentice-Hall, 1999).

30) M. Zhao, W. S. Slaughter, M. Li and S. X. Mao: Acta Mater. 51 (2003) 4461-4469. 